\title{
SPIDAR-if: A Joint User Interface Approach for Hapticised Web Content and Augment Reality Enhancement
}

\author{
Wei-Yu Chen, Makoto Sato, Yen-Ming Chu, and Teruki Honma
}

\begin{abstract}
The paper presents a prototyped jointed control interface approach for understanding hapticised web content and augmented reality (AR) on a work-in-progress instructive platform of haptics (IPOH) over the unreliable network. Our works, in this context, are comprised by two parts. Firstly, we propose a joint user interface SPIDAR-if empowering finger tracking and physical force sense. SPIDAR-if includes a recently notable pocket-sized 3D sensor named "Leap Motion" as an input device and incorporates with another passive 2-degree-of-freedom (DOF) tactile device "SPIDAR" with upturned manipulations in order to truly represent the sense of touch interactions on IPOH system, such as tactile feedbacks derived from in-progressive verbs, HTML components and moving 3D spheres. On the other side, we study the eligibility of SPIDAR-if and its aids with regard to AR world and web content intelligibility in IPOH system which offers the Internet users a bridge to embrace virtual reality by virtue of assisted user interface, wrapping up haptic and web graphical rendering techniques as well as provided virtual environments (VE). Examples of hapticised web content and a 3D AR scenario approaching SPIDAR-if on IPOH with the fingertip are also presented along with preliminary findings.
\end{abstract}

Index Terms-Haptics, SPIDAR, leap-motion, augment reality, web content hapiticisation, haptic data visualization, virtual environment.

\section{INTRODUCTION}

Along with the rapid growth of Internet and web applications, the Internet has brought about overwhelming increments of web data, around 500 terabytes per day [1], and cause people with blindness or partially visual impairments undergo great pain more intensively than before. It urges researchers, developers and engineers to think over how to help them read and understand such large amount of data easier by virtue of information technology approaches. Haptics researchers thus increase its demand on Haptic Data Visualization (HDV) and also address the issues on structural design of relevant platforms. A HDV platform implies to express literal differentiation or/and haptic emotion, and

Manuscript received January 20, 2015; revised August 3, 2015. This work was supported in part by the Riken Research Japan.

Wei-Yu Chen and Makoto Sato are with the Tokyo Institute of Technology, Nagatsuta, Yokohama-shi, Kanagawa-ken, 42592270062 Japan (e-mail: chen.w.ac@m.titech.ac.jp, chen.w.ac@m.titech.ac.jp).

Yen-Ming $\mathrm{Chu}$ is with the National Kaohsiung First University of Science and Technology, Department of Information Management, Taiwan (e-mail: ymchu@nkfust.edu.tw).

Teruki Honma is with the RIKEN Systems and Structural Biology Center, 1-7-22 Suehiro-cho, Tsurumi-ku, Yokohama 230-0045, Japan (e-mail: honma@gsc.riken.jp). encode numerical values, or to say an abstract mathematical concept into cognitive level by providing users both visual and tangible cues [1]-[3]. Take education and online reading as examples: consider that high school students can sense the coherence of velocity to force and experience the resisting change in the object's state of motion physically while their physic teachers are explaining Newton's First Law; people who are visual impaired can also benefit via force mapping of the original value or magnitude whilst they are reading statistical graphs and able to understand the meaning of visualized information concretely through calculated physical forces as hints.

On the other hand, to achieve satisfactory results on HDV, the assisted control device, mainly haptic interface, is equally significant with HDV's platform. As a matter of fact: Haptics, or haptic technology, an immersive technology that enables human being to feel sense of touch, or technically speaking, to acquire realistic experience by means of computers and machines, plays an important role in allowing complex interactions on HDV platform. Initially, Haptics is interpreted as the form of non-verbal body interaction involving touch such as hand-shaking, hugging and kissing, etc. Nowadays this term also refers to capacity of tangible, kinetic and effective expressions [4]. Haptic technology has been studied and commercially available for decades, ranges from hospitals, research institutes, and public space. Previous researches focused on many fields ranging from surgical training in hospital [5], gaming [6] to virtual reality [4]. For examples, to allow doctors to train for simple procedures without endangering patients, to help designers come out their digital clay figures which rely on geometric properties, or to send tactile feels with regarding to inaccessible artifacts to Internet end-users while navigating a museum's website, are exciting instances to illustrate power of Haptics. Nevertheless, current existing haptic interfaces are still facing challenges on HDV for reasons referred as below [7]-[9]. (1) A haptic interface needs to be easily coalesced into current existed HDV platforms. (2) A haptic interface should be low-cost and equipped painlessly without increasing disturbances while users wearing haptic interfaces are sensing hapiticised web content on HDV platform. (3) A haptic interface must be easily deployable for developers and researchers who might not be skilled programming.

This paper contributes on exploiting an advanced control interface SPIDAR-if to the area of HDV where it satisfies with not only upper requirements but also immersive enhancement in VE(s) in light of the proposed approach on a work-in-progress HDV platform named IPOH [2]. The rest of 
the paper is organized as follows: Section II introduces a brief overview of related works. Section III illustrates system architecture and workflow in details. Section IV provides simple experimental examples and early findings. Finally, Section $\mathrm{V}$ concludes the paper and identifies future direction.

\section{RELATED WORK}

In this section, we discuss two components on HDV system: control interface and platform.

\section{A. Choosing Control Interface}

Followed by what we mentioned earlier in this paper, we take use of haptic interface as the control interface rather than using natural user interfaces standalone due to their lack of sense-of-touch effects. In many instances of haptic applications [4]-[6] and HDV [3], Novint Falcon [10], Phantom [11], and CyberGrasp [12] are commonly adopted haptic devices which feature input and output functionalities. These control interfaces enable three-dimensional (3D) and six-degree-of-freedom (6 DOF) force processing, outperform kinesthetic experiences and acquire widely supported by enormous open-source software development kits (SDK) and frameworks. Nevertheless, they might not be affiliated with Internet end-users for reasons, e.g., bulky appearances, burdensome wearing ways and unaffordable price. On the other hand, some studies [3], [13] lighted on another more agile, mouse-assisted passive haptic interface named SPIDAR-Mouse [13], [14], comprised of one USB Mini B cable, one AC Adaptor power supply and a U-shaped plastic frame with one PIC microprocessor embedded, as illustrated in Fig. 1 (a) and (b). SPIDAR [14] is a string-based physical force generation framework with passive force exertion logic, originally invented by Sato Makoto Laboratory (Titech, Japan) and designated for artificial reality. Despite of SPIDAR-Mouse's low DOF, it shows Internet end-users a natural approach and tactile feedback whilst interacting and exploring virtual objects and hapticised web contents.
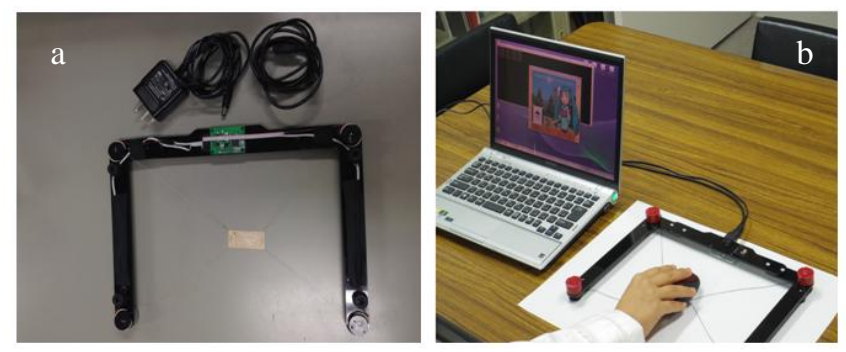

Fig. 1. (a) Physical appearance. (b) User demonstration of manipulation.

It seems to work fine to interact with virtual environments and HDV by means of SPIDAR-Mouse model [2] though, metrics to its properties such as accessibility, reliability, and understandability versus virtual world and web content are far from ideal. To take the modalities of SPIDAR-Mouser farther for HDV, we looked into a latest natural user interface known as Leap Motion and perceived some opportunities. Leap Motion is a recently notable hand sensing controller which envisions human-beings high fidelity user experience on hand tracking and gesture recognition. In the implementation point of view, Leap Motion defines its virtual workspace of cubic 8 feet, a programmable hand skeleton model including fingers, palms, and phalanges for the ease of developing hand motion related applications.

Inspired by Leap Motion's high precision on hand motion data as well as excellent force exertion produced by SPIDAR-Mouse, we herein propose a joint user interface named SPIDAR-if. SPIDAR-if exceptionally projects on advantages of Leap Motion and SPIDAR-Mouse and smothers with imperfections of both. We will discuss it in details in Section III.

\section{B. Platform of Haptic Data Visualization}

The process of HDV is basically considered in the three steps: (1) pre-processing of raw data, (2) abstraction of physics - mathematical values, and (3) Rendering methods in web graphs and haptics [2], see Fig. 2. Bear in mind that any research involved in study on HDV must be clear at what information the graph is about to reveal and what haptic effect is appropriate to present when they are developing a platform of haptics for HDV. For instance, a web user may be intended to find the trend or peak values in graph or perceive the slight difference between two words "swing" and "shake" except for their literal meanings. Under the pre-expected conditions, haptic device shall produce "draw" force and "vibration" force mapping to "trend" and "peaks", respectively.

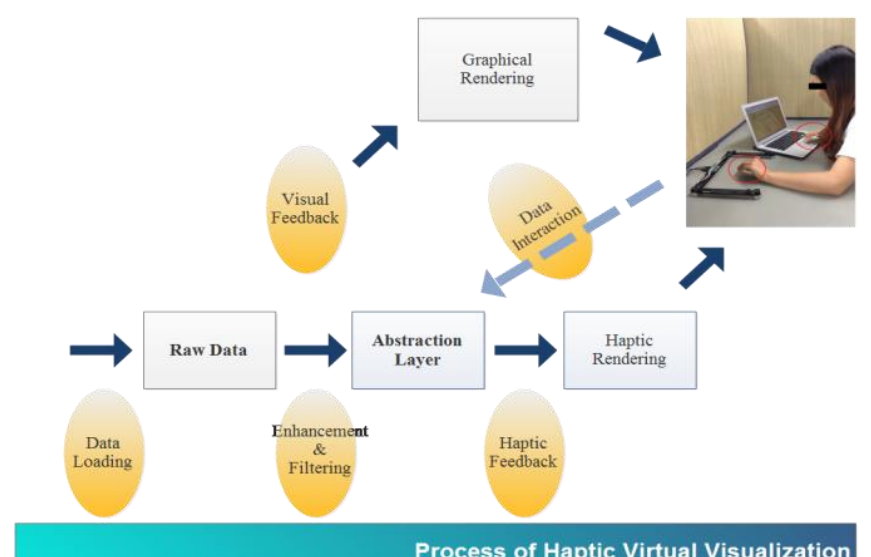

Fig. 2. Process of realizing data visualization and hapticisation.

To satisfy both virtual reality and hapticised web content realization with the processes above for better HDV design, and avert from communication chaos between haptic interface and Internet end-users, an instructive platform IPOH [2] was designated. IPOH is a BDD-oriented (Behavior-Driven Development) platform and benefited on HDV for reasons. (1) It aims to provide people with Internet end-users, especially vision-impaired people, with an ease-of-use haptic experience when they are reading web data on browsers. (2) It classifies haptic effects into three catalogues that can be used as instructive examples to innovate more haptic effects. (3) Different from previous design of web platform using haptic plug-ins and lacking of abstraction layer for HDV, it not merely amends these insufficiencies but also represents good affordance and learnability on HDV. Here we captured two snapshots of the instructive examples in IPOH to demonstrate how it works. The water blue arrows in Fig. 3 indicate the moving paths of the sphere with respect to the verb "shake". Fig. 4 illustrates that during the digital data exploration, users can perceive calculated cued forces when their cursor's 
position is approaching to particular data points in a certain distance and direction on IPOH.

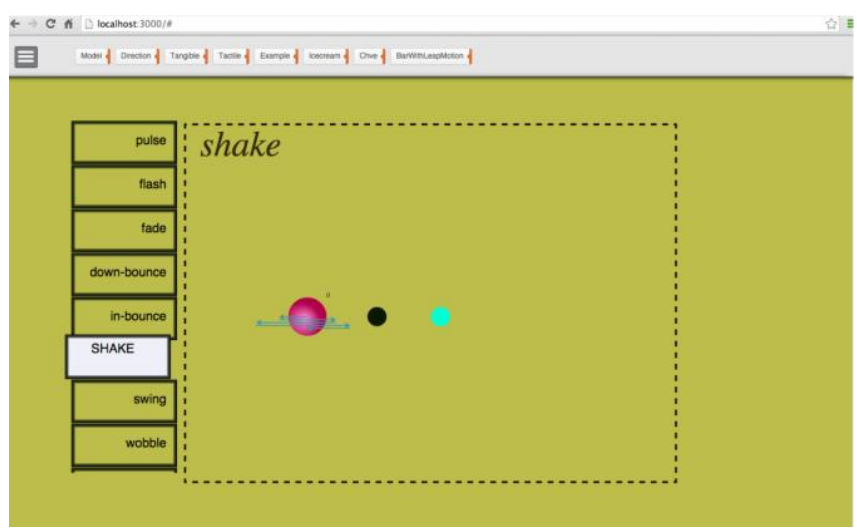

Fig. 3. Catalog one: shake.

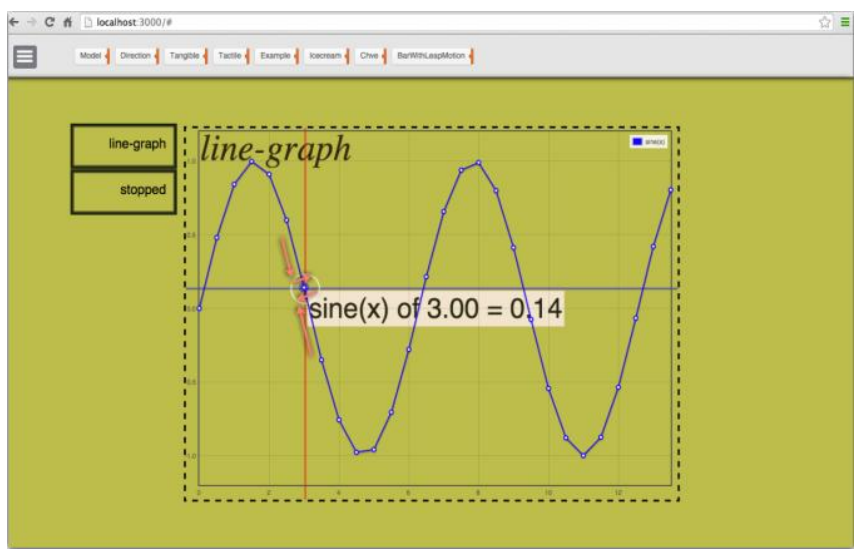

Fig. 4. Digital data exploration and perception.

\section{SYSTEM OVERVIEW}

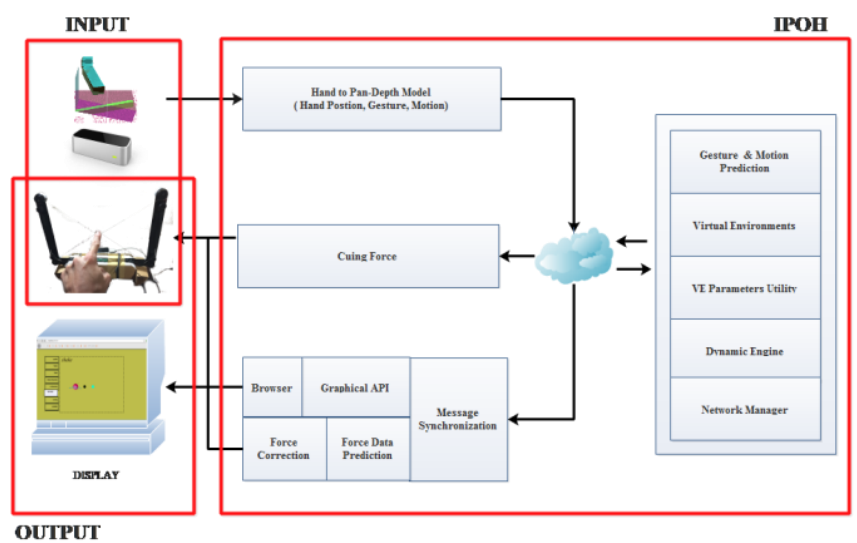

Fig. 5. Input device, output device and HDV platform IPOH.

As illustrated in Fig. 5, the system is comprised of three components: input device, output device and HDV platform. This section describes the system design that conjugates the proposed user interface approach on IPOH platform for better hapticised web content learning and 3D AR experience. Note that in this paper we only focus on design of the proposed user interface and its eligibility on HDV platform, so we are not intended to introduce the topics such as network policy to transfer haptic stream over unreliable network and mechanisms to predict and amend force or/and graphical data. Furthermore, the other to-be-solved issues on gesture recognitions and 3D rendering are not discussed here either, and will be extended to future work. The rest are composed by two sub-sections. In the first sub-section, we will introduce the components in IPOH platform and explain how each of them operates in system. In the other sub-session, we will present the main concept of the proposed user interface approach including implementation details and force generation model.

\section{A. Instructive Platform of Haptics}

To design a stable platform of haptics which supports various web graphical data, information comprehension and simulated haptic effects appropriately can be tricky. IPOH aims at solving three key challenges affecting performance with respect to haptic network platform: communication between electro-mechanical interface and system, representation of hatpic data visualization and haptic data transfer over the unreliable network, see Fig. 5. All browser users and their equipped haptic user interfaces are distributed within wide area network and have individual internet accessibilities. The spirit of data transfer mechanism in this system is addressed as follows. When an event is triggered by an electromechanical interface, it will be detected and mapped into corresponding request, then encoded, segmented into network packets and shipped to the web content server by web application according to current transporting layer policy and network condition. The result will be received, unpacked and decoded through haptic application to electro-mechanical interface to conduct correct effects. IPOH specializes on the update rate issues with respect to three challenges in haptic system and addresses their restrictions in order to display the visual feedback and reinforce the feeling of sense-of-touch, i.e., $20-30 \mathrm{~Hz}$ for continuous graphic rendering, $1000 \mathrm{~Hz}$ for haptic update rate and less than $60 \mathrm{~ms}$ in network latency.

\section{B. SPIDAR-if}

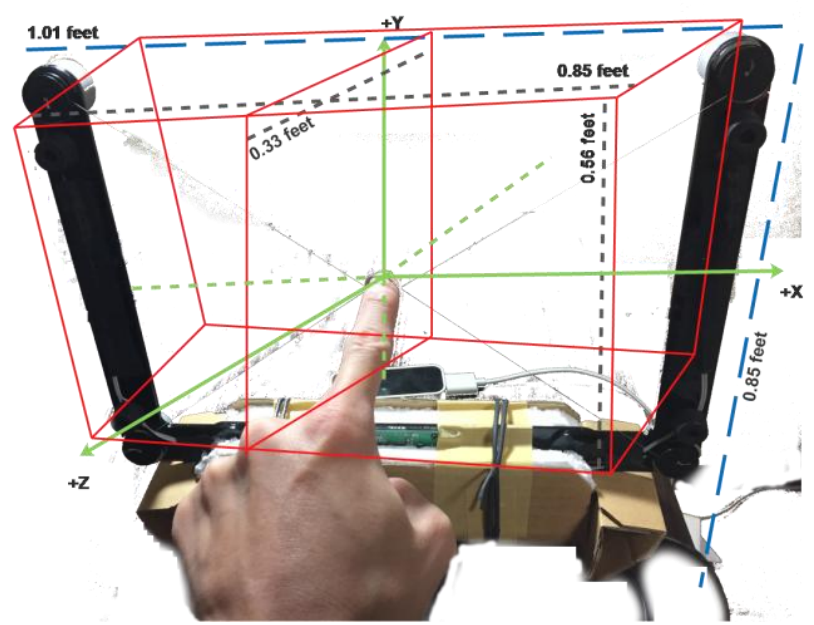

Fig. 6. Spatial representation of SPIDAR-if workspace.

SPIDAR-if is a joint control interface combined with an IR emitters and CCD cameras embedded controller Leap Motion, and a string-based passive electromechanical device SPIDAR composed by a U-shaped frame and 4 motors, see Fig. 6 and Fig. 7. As shown in Fig. 6, the model of SPIDAR-if differs from SPIDAR-Mouse's (Fig. 1) in both their appearance and manipulation. At first, we provide a corrugated cardboard 
pedestal as a support to avoid from using SPIDAR-Mouse flat-wise. Secondly, we replace mouse-centralized with fine wire encircled index finger. Finally, same with Leap Motion, we also employ a right-hand Cartesian coordinate system, construct a 3D coordinate geometry with size cubic 0.16 feet and target origin on the geometric center of SPIDAR-if's frame. All behaviors and gestures of SPIDAR-if are conducted by an index finger, e.g., finger position, finger bends, finger taps as well as hand skeleton information including palms and phalanges, are recognized and traced by Leap Motion, and reported to system for advanced processing. Remind that SPIDAR-if considers only one single finger tracing model and does not enable complex multi-finger recognition which Leap Motion supports though.

The following formula explicitly describes applied force generation algorithm on finger-force model:

$$
\begin{gathered}
F_{n}(x, y, \partial, t)=\sum_{i=1}^{N} \kappa \cdot \delta_{\mathrm{XY} \hat{Z}, n}(t)+W \cdot S_{\mathrm{XY} \hat{Z}}\left(t_{\alpha}, t_{\beta}\right) \\
\delta_{\mathrm{XYZ̃}}(n)=\left(P_{\mathrm{XYZ}}(n)-P_{\mathrm{XYZ}}(n-1)\right) / P_{\mathrm{XYZ}}(n-1)
\end{gathered}
$$

where $k$ is the elastic constant with respect to each dynamic object and derived from their weight, size and dynamics in virtual world. $\mathrm{W}$ is represented as a coefficient function of SPIDAR-if in geometric coordinate $S . S_{x, y, z}$ is the time-varied index finger position data in which $\hat{z}$ is indicated as a selective vector. We apply a selective vector on Z-plane as a cue for enhancing visual effect because it is not truly treated on SPIDAR-if's workspace. In addition, we measure start time $t_{\alpha}$ and end time $t_{\beta}$ of the fingertip movement during certain time period in support of hand gesture tracking. A simple example of simulated force result by using (1) and (2) is clearly illustrated that during the digital data exploration, users can perceive calculated cued forces when their cursor's position is approaching to particular data points in a certain distance and direction on IPOH in Fig. 7

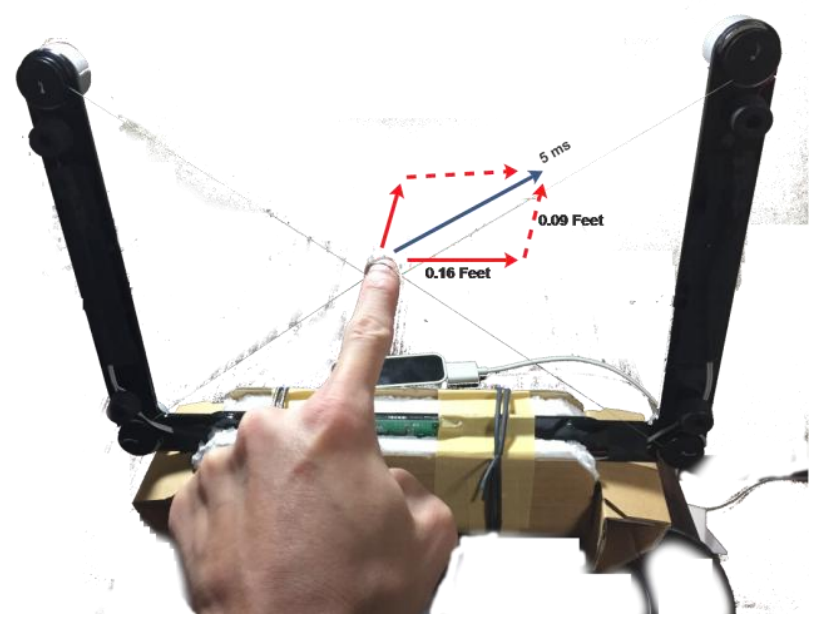

Fig. 7. An illustration of manipulating SPIDAR-if.

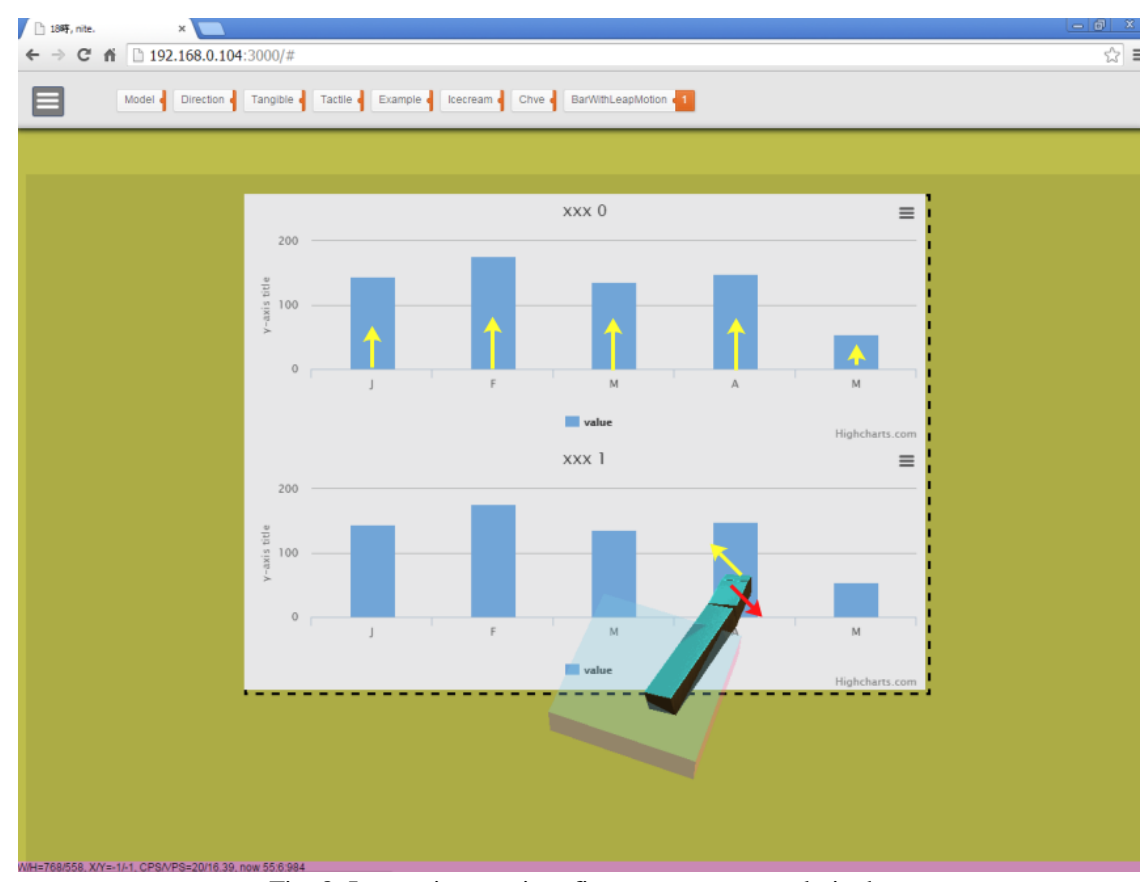

Fig. 8. Interactive sensing: finger movement and pinch.

\section{PRELIMINARY EXPERIMENT}

In this section, we present two conceptual examples of applications to our work-in-progress research. The primary experiments are conducted through a set of physical operations and observations in order to confirm eligibility and assistance of SPIDAR-if on HDV and AR. Same as shown in Fig. 6, we place Leap Motion in front of SPIDAR-if and keep it flat. An active interactive space is thus defined once geometrical location of SPIDAR-if is fixed. The tasks are illustrated in Fig. 8 and Fig. 9 and described as below. Charts are normally simple tools to visualize digital information on web pages. Through mapping the "punch" to bars on a graph and combining the interchange among the other visualized content along with a haptic pulling feedback shown in Fig. 8, it becomes more effective and understandable than browsing alone; similarly, Fig. 9 shows a visual example of an AR 3D scenario: a moving fingertip in geometrical space is approaching a falling virtual sphere and at the moment when they approach, a circle motion conducted by fingertip is identified and lead the upper sphere's flung-off. In the above 
instances not only reachable and touchable but also physically employable interactions with falling virtual spheres are realized by means of Leap Motion controller, SPIDAR and haptic rendering algorithm.

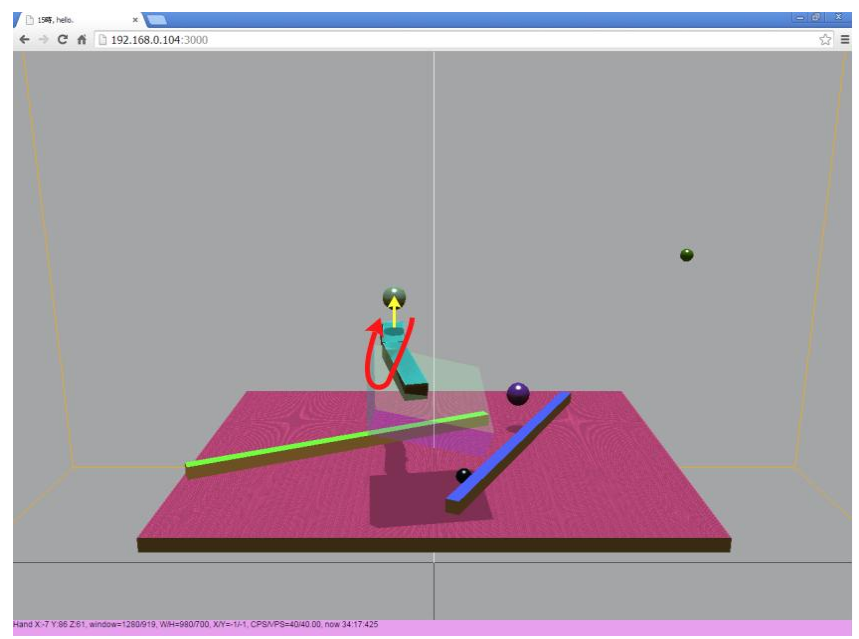

Fig. 9. An example of an AR 3D application.

\section{CONCLUSION}

Challenges are emerged from designing user interface for Internet end-users to help understand web content and enhance interactivity in the AR world. In this paper, a prototypical implementation of joint user interface approach combines with Leap Motion and SPIDAR, and supports interaction of AR world and normal web-pages is proposed. SPIDAR-if allows for mutually interacting with web content and even manipulating them based on finger-force model. The proposed approach was derived from SPIDAR-Mouse primarily, but we further extend its appearance and manipulations for advanced use. In addition, a work-in-progress haptic data visualization platform IPOH is addressed to exploit how a HDV system works, e.g., communications protocols between user interfaces and a haptic system, mathematics-to-physics mapping and haptic data transfer over the physical network. Two conceptual examples of haptic applications shown in section IV, moreover, indicate the eligibility of SPIDAR-if and its aid on understandability of web content. In particular, by incorporating with Leap Motion Controller, haptic experience by conducting our approach is obviously amplified on IPOH. Nevertheless, the proposed approach has limitations: restricted web content support, imperfect force exertion and inaccurate gesture recognition. Recent studies on HDV suggest that support of more understandability on different catalogs of web content such as map, network and diagram, and fulfillment of enhancing user experience on AR applications and those imply the issues on complex HDV and accuracy of finger-force model remains. Although our current work focus on implementing eligible user interface, with the increasing distributions in a classic haptic platform, other environments and modalities are also interesting for future research.

\section{REFERENCES}

[1] A. Cichocki and R. Unbehaven, Neural Networks for Optimization and Signal Processing, 1st ed. Chichester, U.K.: Wiley, 1993, ch. 2, pp. 45-47.
[2] S. Paneels and J. C. Roberts, "Review of designs for haptic data visualization," IEEE Transactions on Haptic, vol. 3, no. 2, pp. 119-137, 2010.

[3] W. Y. Chen and M. Sato, "An applicable platform for web interactive content hapticisation," presented in International Conference on Civil, Materials and Computing Engineering, Tai-Chung, Taiwan, December 6-7, 2014

[4] K. Moustakas et al., "Haptic rendering of visual data for the visually impaired," IEEE MultiMedia, vol. 14, no. 1, pp. 62-72, 2007.

[5] M. A. Srinivasan and C. Basdogan, "Haptics in virtual environments: Taxonomy, research status, and challenges," Computers \& Graphics, vol. 21, no. 4, pp. 393-404, 1997.

[6] B. Cagatay et al., "Haptics in minimally invasive surgical simulation and training," Computer Graphics and Applications, vol. 24, no. 2, pp. 56-64, Feb. 2004.

[7] J. Wood et al., "The design and evaluation of a computer game for the blind in the GRAB haptic audio virtual environment," in Proc. Eurohpatics, 2003, pp. 148-158.

[8] E. Ruffaldi et al., "A haptic toolkit for the development of immersive and web-enabled games," in Proc. the ACM Symposium on Virtual Reality Software and Technology, 2006, pp. 320-323.

[9] Y. Seo, D. Y. Kim, and S. H. Suh, "Development of web-based CAM system," The International Journal of Advanced Manufacturing Technology, vol. 28, pp. 101-108, Feb. 2006.

[10] H3D API. [Online]. Available: http://www.h3dapi.org

[11] S. Martin and N. Hillier, "Characterisation of the Novint Falcon haptic device for application as a robot manipulator," presented at Australasian Conference on Robotics and Automation (ACRA), Sydney, Australia, December 2-4, 2009.

[12] T. H. Massie and J. K. Salisbury, "The phantom haptic interface: A device for probing virtual objects," in Proc. the ASME Winter Annual Meeting, Symposium on Haptic Interfaces for Virtual Environment and Teleoperator System, vol. 55, no. 1, pp. 295-300, Nov. 1994.

[13] C. Magnusson, K. Rassmus-Gröhn, C. Sjöström, and H. Danielsson, "Navigation and recognition in complex haptic virtual environments-reports from an extensive study with blind users," presented at Euro Haptics, Edinburgh, UK, July 8-10, 2002.

[14] K. Asai, N. Takasa, and M. Sato, "2D force feedback for haptic interaction with SPIDAR-mouse by mapping 3D surface to plane in molecular docking," presented at IEEE World Haptics Conference, Daejeon, Korea, April 14-18, 2013

[15] M. Sato, "Spidar and virtual reality," in Proc. the 5th Biannual World Automation Congress, 2002, pp. 17-23.

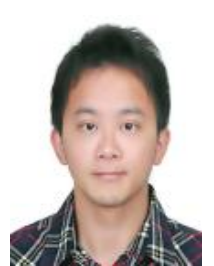

Wei-Yu Chen is a PhD student at the Department of Artificial Intelligent and System Science, Tokyo Institute of Technology, Tokyo, Japan, and a research associate in RIKEN, Japan. He received his BSc degree in 2008 in computer science and the MSc degree in 2010 in communication engineering from National Tsing-Hua University, HsingChu, Taiwan. His main research interests include virtual reality, haptics, embedded system and collaborative network analysis.

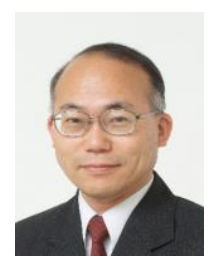

Makoto Sato was born on February 26, 1951. He received his B.E., M.E. and Dr. degrees in 1973, 1975 and 1978, respectively, from Tokyo Institute of Technology. From 1978 to 1986 , he was a research associate in the Department of Computer Science, Tokyo Institute of Technology. He was appointed to be an associate professor in 1986 and a professor in 1996 at Precision and Intelligence Laboratory, Tokyo Institute of Technology. He has worked on pattern recognition, computer vision, human interface and virtual reality. He is a member of IEICE, ITE, IPSJ, VRSJ and IEEE.

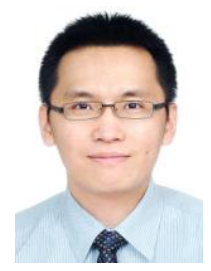

Yen-Ming Chu received the $\mathrm{PhD}$ degree in communications engineering from National Tsing-Hua University, Taiwan, in 2010. From 2001 to 2005, he served as an assistant researcher in Telecommunication Labs of Chunghwa Telecom Co. Ltd. and worked in NetXtream Corp. from 2008 to 2010. Since August 2014, he has been with the Department of Information Management, National Kaohsiung First University of Science and Technology, Kaohsiung, Taiwan, where he is an assistant 
professor. His current research interests include gamification, multimedia networking, and network science.

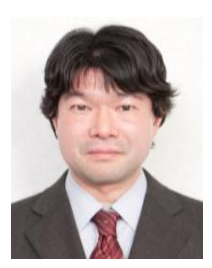

Teruki Honma was born in Hokkaido prefecture, Japan, in 1969. He graduated from Faculty of Science, Hokkaido University in 1991 for natural product chemistry supervised by Prof. Murai. After graduation of Graduate School of Hokkaido University in 1993, he obtained the Ph.D. in 2001 for drug design and synthesis of potent anticancer agents. In 1993, he joined Banyu Pharmaceutical Co., Ltd and had been in charge of drug design and synthesis of anticancer agents, anti-coagulant agents, anti-diabetes agents. In 2004, he moved to Pfizer Japan Inc. as a principal scientist and promoted to the leader of computational chemistry group. After site closing of Pfizer research institute in Japan, he moved RIKEN (Yokohama city, Kanagawa prefecture) as the team leader of Structure-based Molecular Design Team. His majors are computational science especially new technology development of drug design and its applications to real drug discovery targets. Now he participates in national projects ("Platform for Drug Discovery, Informatics, and Structural Life Science" and "Drug Discovery Network") of drug discovery in Japan. 